\title{
MRI AND HIGH RESOLUTION CT IN CONGENITAL HEARING LOSS
}

\author{
Ahmed A. M. Abd Alla ${ }^{1}$, Ibrahim A. Lebda ${ }^{1}$, Sonia Y. Mohamed ${ }^{1}$, Ezzat A. Merwad ${ }^{2}$ \\ ${ }^{1}$ Department of Radiology, Faculty of medicine, Zagazig University. \\ ${ }^{2}$ Department of Otolaryngology, head and neck surgery, Faculty of medicine, Zagazig University.
}

\begin{abstract}
Purpose: The aim of this study is to assess the role of Magnetic resonance imaging (MRI) and high resolution CT (HRCT) in patients with congenital hearing loss.

Patients and methods: Sixty patients who having congenital hearing loss (CHL) will be included in our study. Full history taking, clinical examination, Oto-laryngology examination and audiological evaluation tests, MRI and non-enhanced HRCT.
\end{abstract}

Results : The results of this study cannot be analysed from an epidemiological point of view due to the absence of a comprehensive repeated investigation, but they illustrate and clearly justify the need to perform a standardized aetiological work-up. HRCT was superior in detection of bony landmarks of the temporal bones. These findings were the shape and orientation of the petro-temporal bones, the patency of the external auditory canal (bony atresia), associated tympanic cavity and ossicular chain anomaly. MRI of the both temporal bones were accurate in detection of abnormalities in the membranous labyrinth and inner ear malformations. The use of balanced-Fast Field Echo (b-FFE) series on the internal auditory canals to evaluate the cochlear nerve. Our MRI studies were unable to evaluate the condition of the middle ear and mastoid cavity, which appears as intermediate and bright signals seen in both T1- and T2-weighted images. However, HRCT clearly shows the condition of the bone cavity and bone marrow of the tympanic cavity, and the pneumatization of the mastoid process if present.

Conclusion : Imaging plays an important role in diagnosis and work-up of patients with congenital hearing loss. There are a whole lot of etiologies, many of which have typical findings on HRCT and MRI, which help in differentiation of these causes and therefore guides the treating surgeon in further management. Both HRCT and MRI are complimentary to each other and should be judiciously used by the radiologist in diagnosing the various congenital malformations.

Key Indexing Terms: Congenital Hearing Loss (CHL), Magnetic Resonance Image (MRI), High Resolution CT (HRCT), decibels (dB), pure tone average (PTA), Hertz (Hz), minimal alveolar concentration (MAC),

Tesla (T), balanced-Fast Field Echo (b-FFE), Maximum intensity projection (MIP), multi-planar reformat (MPR).

\section{INTRODUCTION}

$\mathbf{H}$ earing dysfunction is the most common sensory deficit in children. If hearing is lost, especially in the postnatal period or during infancy, it may have a huge impact on life, interfering with the acquisition of spoken language and social development. It is therefore important to detect any abnormalities at a very early age ${ }^{(1)}$. The detection of hearing loss at an early stage is vital to the child's development. In children with profound bilateral congenital hearing loss, delayed neural auditory development may occur. If the problem is left beyond the age of 5 years, it is possible that the child may never develop good functional hearing. This is problematic in two ways: first, the child will never develop aidable hearing and will therefore be dependent on sign language, and second, the child will not develop spoken language ${ }^{(2),(3)}$.

Sensorineural hearing loss can be caused by lesions anywhere along the auditory pathways, from the cochlea to the central nervous system. Etiology of sensorineural hearing loss (SNHL) is diverse and includes genetic defects, and labyrinthine malformations ${ }^{(4)}$.

\section{AIM OF THE WORK}

The aim of this study is to assess the role of high resolution CT and MRI in patients with congenital hearing loss.

\section{PATIENTS AND METHODS}

Sixty patients who having congenital hearing loss, included in our study; (35) males and (25) females, their age ranged from $2 \mathrm{~ms}$ to 19 years with mean age of 3.57 years, with $\mathrm{SD}=$ 3.07, referred from Department of Otolaryngology, Head and Neck surgery, Audiology unit and out-patient clinics at the Zagazig University Hospitals. We obtain an institutional review board approval from our hospital, explain the procedures to the patient or the parents and an informed consent from the parents or relative are done to all our patients before examination. Clinical records of each patient was identified and analysed regarding the age at onset of HL, pattern, type and course of HL. All available of audiometric and vestibular findings were studied. 
Patients subjected to :-

1- Full history taking and clinical examination.

2- Otolaryngotoic examination and audiological evaluation tests.

3- Non-enhanced HRCT.

4- Magnetic resonance imaging.

Audiological evaluation tests:-

- All paediatric patients are evaluated by using a forum of BASIC AUDIOLGICAL EVALUATION FOR CHILDREN and in two cases an AUDIOLGICAL EVALUTION FOR ADULT were completed at the Audiology Unit in Zagazig University Hospitals.

- The frequency or pitch of the sound is referred to in Hertz $(\mathrm{Hz})$. The intensity or loudness of the sound is measured in decibels $(\mathrm{dB})$. The responses are recorded on a chart called an audiogram that shows intensity levels for each frequency tested.

All patients were examined in each ear and the degree of hearing loss is measured in decibels (dB) and further characterized into, normal hearing loss ranges from $0-15 \mathrm{~dB}$, a minimal hearing loss ranges from $16-25 \mathrm{~dB}$, a mild hearing loss ranges from $26 \mathrm{~dB}$ to $40 \mathrm{~dB}$, moderate from 41 to $55 \mathrm{~dB}$, moderately severe from 56 to 70 , severe 71 to $90 \mathrm{~dB}$, and a profound hearing loss > $90 \mathrm{~dB}$ HL. The air conduction testing, using speakers and headphones and the bone conduction testing is performed by the use of a vibrator positioned behind the ear on the mastoid or on the forehead. The threshold is determined at the lowest sound level that the child can hear the tone $50 \%$ of the time. Results are plotted on a graph called an audiogram, and a pure tone average (PTA) is determined from the thresholds at 250, 500, 1000, 2000, 4000 and $8000 \mathrm{~Hz}$.

- Conductive hearing loss is considered when, air conduction thresholds are abnormal, bone conduction thresholds are normal, and an air-bone gap is present.

- In sensorineural hearing losses, air conduction and bone conduction thresholds are both abnormal, but are impaired to approximately the same degree (no airbone gap present). In mixed hearing losses, air conduction and bone conduction thresholds are both abnormal, but air conduction threshold are worse than bone conduction thresholds (an air-bone gap is present).

- The data on the tympanogram are categorized as either a Type A, B, or C. Type A refers to eardrum movement within normal limits. Type B indicates little or no eardrum movement suggesting fluid in the middle ear space. Type $\mathrm{C}$ refers to a middle ear with negative pressure and considered borderline normal.

\section{Preparation of the patient:}

Patients examined were fasting at least six hours prior the examination and external ear aids or earrings in female patients are removed.

\section{Sedation and general anaesthesia in uncooperative children}

Sedation using Chloral Hydrate were used in a dose of $50 \mathrm{mg} / \mathrm{kg}$ body weight given orally 20 to 30 minutes before examination. Some children needed additional sedation by intravenous diazepam (Valium) in a dose of $10 \mathrm{mg}$. All patient were examined by our colleague anesthetist and monitored while doing the CT scanning. General anesthesia also needed by Fluthane ventilation with minimal alveolar concentration (MAC) of $1.8 \%$ and added:-

Atropine in a dose of $1 \mathrm{mg} / \mathrm{kg}$.

Ketamine in a dose of (1) $\mathrm{cm}$.

\section{High resolution computerized tomography:-}

The patients were subjected to CT scanning done in all cases using (General electric CT) machine (GE High Speed Medical system, USA) and Dual-slice helical CT imaging (GE Medical system, USA).

\section{A) Performance of scanning:}

Axial scanning:- The patient was supine in position, lateral scanogram (scout view) is taken. The reference lines are drawn parallel to the supra-orbito-meatal line baseline, making $30^{\circ}$ with the orbito-meatal base line, thus reducing the irradiation to the lens of the eyes to the least possible dose of radiation. Axial slice angle was 0 degree, where at this angle, better quality of the reformatted images were achieved.

Coronal scanning:- The patient lies supine with the head hyperextended being depended off the edge of the CT table with supporting the patient head to avoid hyperextension. A lateral scanogram (scout view) is taken and localization lines are plotted perpendicular to the orbitomeatal base line aided by gantry angulation of 2025 degrees.

Image processing: Coronal and oblique reformates was done from the axial images. The muliplaner reformates (MPR) images in coronal view was done aligned in perpendicular axis to the, and the oblique view was done both aligned in parallel axis and perpendicular to the line drawn along the superior semicircular canal. Temporal bone CT was performed with a singledetector CT scanner (SDCT) and Two detectorrow CT scanner (MDCT). Images were reconstructed using a high spatial resolution bone algorithm with a slice thickness of $1 \mathrm{~mm}$, a field of view (FOV) of $80 \mathrm{~mm}$, and a matrix of $256 \times$ $256(\mathrm{SDCT})$ or $512 \times 512$ (MDCT). The 
reformations for the short axis (Poschl) projection for the cochlea by drawing a line passing through the upper superior semicircular canals and reformate image parallel to this line can produce this projection. The orientation of the reformations for the long axis (Stenvers) projection by creating a small stack perpendicular to the short axis series to create these projection.

Magnetic Resonance Imaging Examination:-

All the MR images were performed by using Philips Achieva 1.5 T, Netherlands, at the Faculty of medicine, Zagazig university hospitals. Type of coils used:

SENSE Head phased-array coil (8) :- Panel (1)

1. The head coil is placed on the tabletop.

2. The mattress is placed on the table top and we make sure the head support is secure.

3. The patient head is placed in the head support.

o The head fixation strap for extra immobilization.

o The small wedges to immobilize the head by placing them firmly between the head and the sides of the support.

4. We pull the sliding part gently over the head and face.
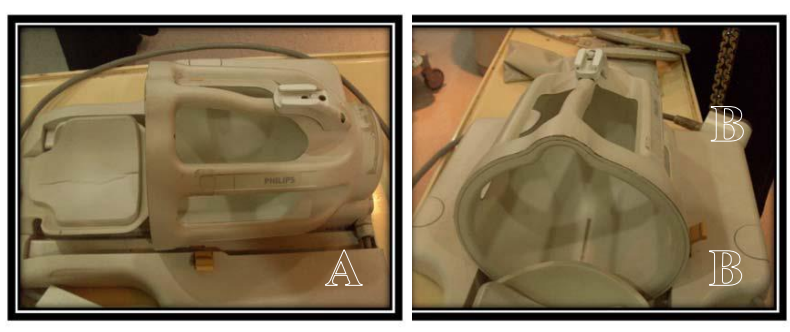

Panel (1) : (A) and (B) Sense head phased-array coil (8).

\section{Patient observation via Sensors}

Consumable items for physiology e.g. sensors and cables for respiratory and VCGtriggering (Vector-Cardio-Graphy) are arranged before set of the patient. The Physiology signals are rescaled via the rescale signal function in the Physiology window.

Optimal image quality for imaging: To increased image quality we require a small field of view (FOV), we typically use $1.5-2.5 \mathrm{~cm}$ FOV for examinations. The image matrix should be 205 to 256. One to $2 \mathrm{~mm}$ slice sections and slice gap about $10-20 \%$ of slice thickness $0.1-0.5 \mathrm{~mm}$.

The Fast Field Echo (FFE) :-

The FFE sequence is a gradient echo pulse sequence The FFE sequence is a gradient echo pulse sequence with a gradient read-out. A $180^{\circ}$ refocussing pulse will not be applied in this sequence, consist of a number of successive RF pulses. Stimulated spin echoes are also generated, as always when there is more than one RF pulse. This is especially true for short TR's. Several FFE techniques are available which can be selected via the parameter 'Contrast enhancement'.

\section{Extended MR Workspace :-}

The data released as multi-frame MR DICOM standard are transferred into an Extended MR Workspace, where the images are processing via a workstation software package designed to run on standard PC hardware. The hardware required is made up of "off-the-shelf" standard computer components, Extended MR Workspace using (Extended MR Workspace R2.6.3.1) performs viewing, image manipulation, communication, printing, and quantification of images and the images were processed using MIP, volume rendering, or 3D navigator techniques.

\section{RESULTS}

This study included 60 patients; (35) males and (25) females, their age ranged from 2 ms to 19 years with mean age of 3.57 years, $\mathrm{SD}=$ 3.07, referred from Department of Otolaryngology, Head and Neck surgery, Department of Audiology and out-patient clinics at the Zagazig University Hospitals.

As regarding the type of congenital hearing loss, the patient's final data diagnosis from clinical evaluation, audiometric studies with HRCT and MRI examinations are collected. The (32) patients with Congenital Conductive Hearing Loss (CCHL) were the most common in our study (53.33 \%), (23) patients with Congenital Sensineural Hearing Loss (CSHL) representing $(38.3 \%)$ and (5) patients with Mixed Congenital Hearing Loss (MCHL) representing (8.33 \%). Clinical presentations of hearing loss are summarized in Chart (1).

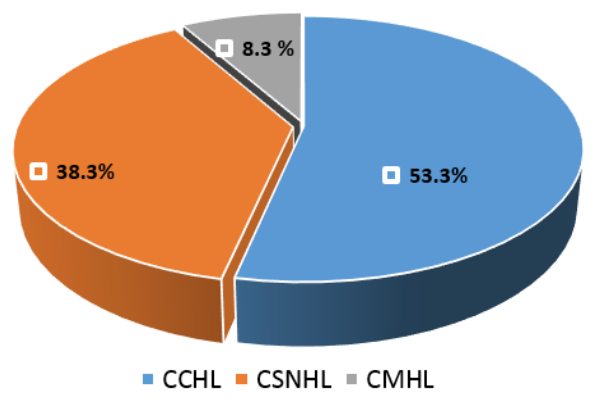

Chart (1): Pie chart demonstrates the percentage of the types of congenital hearing loss. Congenital conductive hearing loss (CCHL), congenital sensineural hearing loss (CSNHL) and congenital mixed hearing loss (CMHL).

The final diagnosis of the (60) patients (35 males and 25 females), included in our study, according to HRCT and MRI results as well as 
clinical evaluation and audiological assessment. Fourteen cases of them (8 males and 6 females) evaluated as candidate for cochlear implantation, (4) cases ( 2 males and 2 females) are further needed follow as illustrated in Table (1)

\section{Conductive hearing loss:-}

Thirty two patients were diagnosed as conductive hearing loss. They were (22 males and 10 females), their age ranged from 2 months to 19 years with mean age of 4.1 years and $\mathrm{SD}=3.844$.

\section{(A) Anomalies of the external ear: - (Case 1)}

Fourteen patients were diagnosed as having external ear anomalies, they were (8) males and (6) females and their age ranged from
$2 \mathrm{~ms}$ - 19 years. They are all having unilateral complain.

- Patients with malformations of the outer ear graded according to the severity of malformations into:

2 patients (both are males with age 3 and 4 years) with minor auricular malformations: small malformations of the cartilage of the auricle with good pneumatization of the middle ear and mastoid.

- 4 patients ( 2 males and 2 females with age range from $2 \mathrm{~ms}$ to 19 years) with middle grade auricular malformations: microtia of the auricle, bony atresia of the outer ear canal, normal or poor pneumatization of the middle ear and mastoid.

Table (1): The final radiological diagnosis of the patients with congenital hearing loss.

\begin{tabular}{|c|c|c|c|c|}
\hline Final diagnosis & Male & Femal & Total & Percent \\
\hline A) Congenital Conductive Hearing Loss & 22 & 10 & 32 & $53.33 \%$ \\
\hline $\begin{array}{l}\text { I. Anomalies of the external ear } \\
\text { Auricle } \\
\text { EAC bony atresia }\end{array}$ & $\begin{array}{l}8 \\
8 \\
\end{array}$ & $\begin{array}{l}6 \\
6 \\
\end{array}$ & 14 & $23.33 \%$ \\
\hline $\begin{array}{l}\text { II. Anomalies of the middle ear } \\
\text { Tympanic cavity } \\
\text { Ossicular chain } \\
\text { Congenital Cholesteatoma }\end{array}$ & $\begin{array}{l}6 \\
9 \\
2\end{array}$ & $\begin{array}{l}2 \\
4 \\
1\end{array}$ & 18 & $30 \%$ \\
\hline $\begin{array}{l}\text { B) Congenital Sensorineural Hearing Loss } \\
\text { Normal cochlea and inner ear structures } \\
\text { Cochlear Abnormalities } \\
\text { Cochlear Nerve Hypoplasia } \\
\text { Enlarged Vestibular Aqueduct } \\
\text { Malformations of the Vestibule and Semicircular Canals } \\
\text { IAC Anomalies } \\
\text { Sundromic hearing impairment }\end{array}$ & $\begin{array}{l}14 \\
8 \\
1 \\
2 \\
5 \\
2 \\
1 \\
2\end{array}$ & $\begin{array}{l}9 \\
2 \\
1 \\
3 \\
3 \\
2 \\
1 \\
1\end{array}$ & 23 & $38.33 \%$ \\
\hline C) Mixed Hearing Loss & 3 & 2 & 5 & $8.3 \%$ \\
\hline
\end{tabular}

- 8 patients (4 males and 4 females with age range from 1 year to 5 years) with severe auricular malformations: microtia of the auricle, bony atresia of the external auditory canal, poor or absent pneumatization of the middle ear cavity or the mastoid and associated middle ear ossicular bones malformations.

\section{(B) Middle ear cavity: - (Case 2)}

In our study, (18) patients diagnosed as having middle ear anomalies, their age ranged from 1 year to 19 years and the main complaints was hearing loss; (6) patients with abnormal tympanic cavity (4 males and 2 females), (13) patients with anomalies of the ossicular chains (9 males and 4 females) and (3) patients with congenital cholestatoma (2 males and one female).

According to HRCT diagnosis, as seen in Table (2) and Chart (2), the tympanic cavity was within normal limits in (10) patients (7 males and 3 females), hypo-plasia of the tympanic cavity in (6) patients (4 males and 2 females) and one case with oval window bony atresia. The ossicular chain anomalies were the most common encountered in our study (13) patients (9 males and 4 females), the incudo-malleolar fusion seen in (5) patients ( 3 males and 2 females), tympanic fixation (2) male patients, displacement with epitymapnic fixation (4) patients (2 males and 2 females), incudo-malleolar disarticulation in (2) females. The mastoid bones pneumatization were normal in (6) patients, poor pneumatiztion in (4) patients and absent pneumatization in (3) patients. Three patients ( 2 males and a female) diagnosed having middle ear congenital cholesteatoma, seen as non-dependent soft tissue density mass seen partially filling the middle ear cavity, with intact ossicular chain and no evidence of bone erosion in all cases. Two operated male patients and the 
female patient had left microtia and external canal bony atresia and schedule for operation.

MRI examination, for (8) patients to the middle ear malformation and additional inner ear abnormalities, the abnormalities detected divided into four groups: group I, middle ear cavity and mastoid cavity type (abnormal intensity of the middle ear cavity and mastoid cavity) these include (3) patients; group II, middle ear cavity type (abnormal intensity of the middle ear cavity) in one patient; group III, mastoid cavity type (abnormal intensity of the mastoid cavity in (2) patients and finally in group IV had no abnormality of the middle ear cavity or mastoid cavity in (2) patients.

Table (2): HRCT appearance of the anomalies of middle ear in (18) patients.

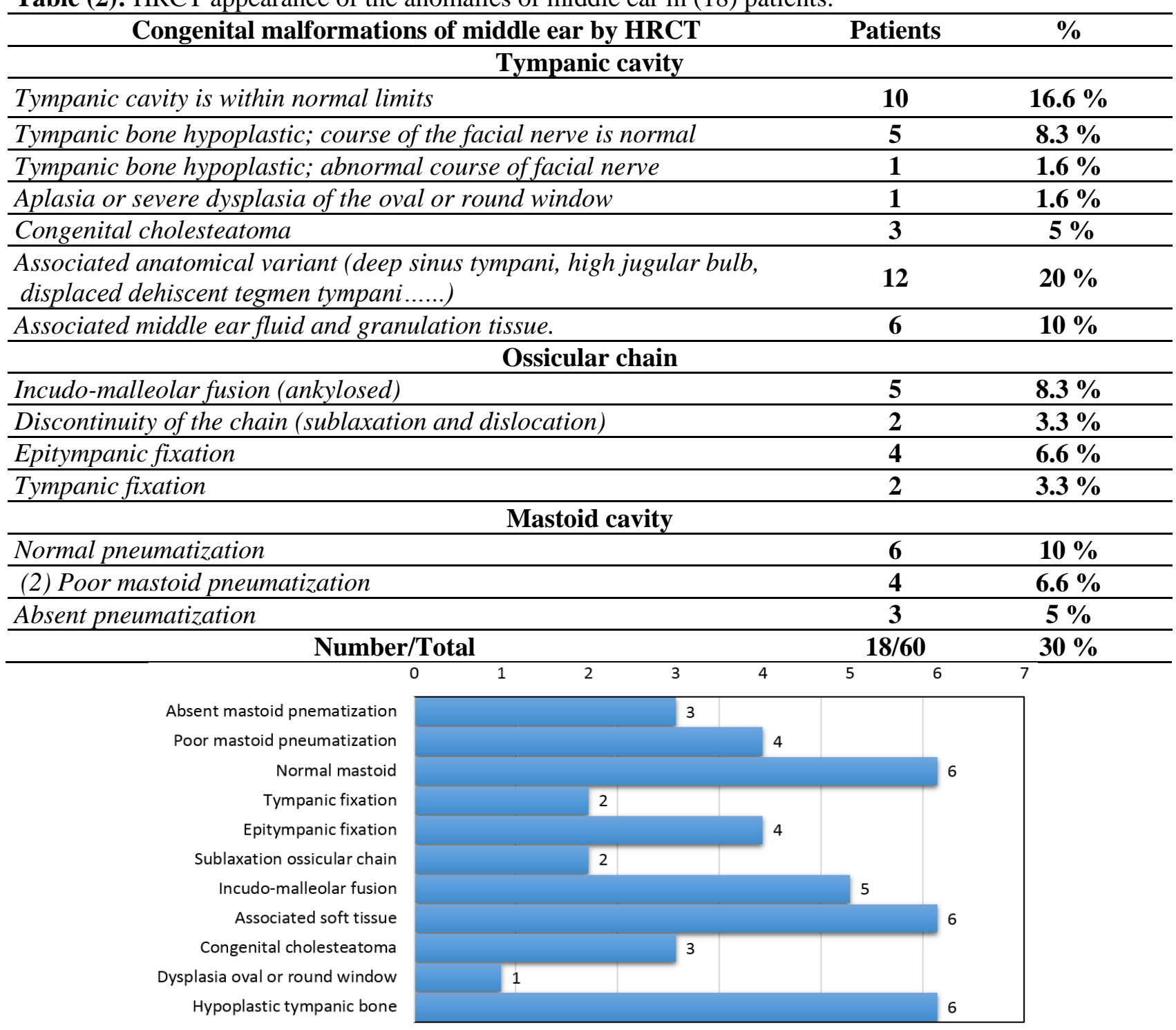

Chart (2): Column chart in HRCT findings of anomalies of the middle ear in (18) patients. 


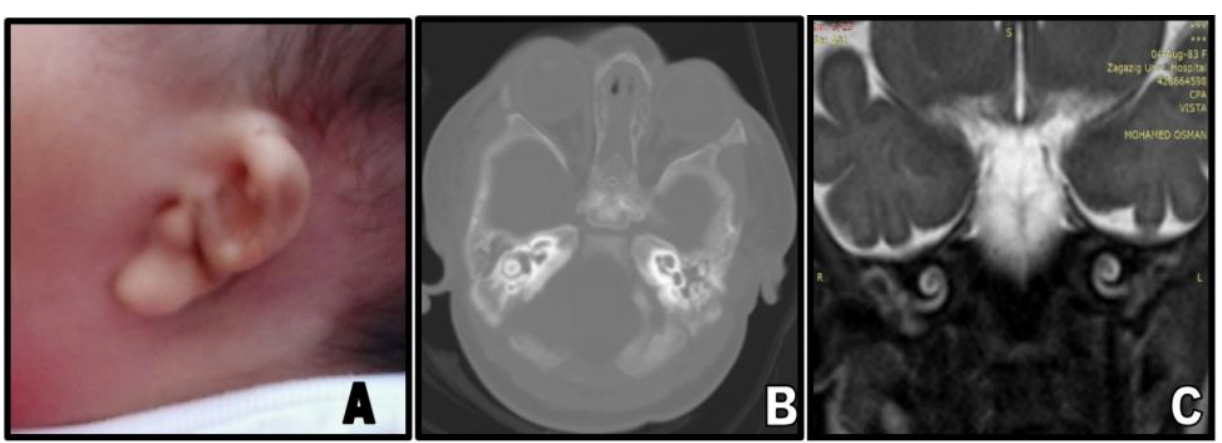

Case (1): A 2 months old patient with congenital malformed left ear pinna (A) A photograph of the degree of the malformed auricle. (B) Axial HRCT of the both temporal bones of with left external auditory canal bony atresia. (C) The coronal b-FFE of the both inner ears, demonstrate normal cochlear turns and other inner ear structures.
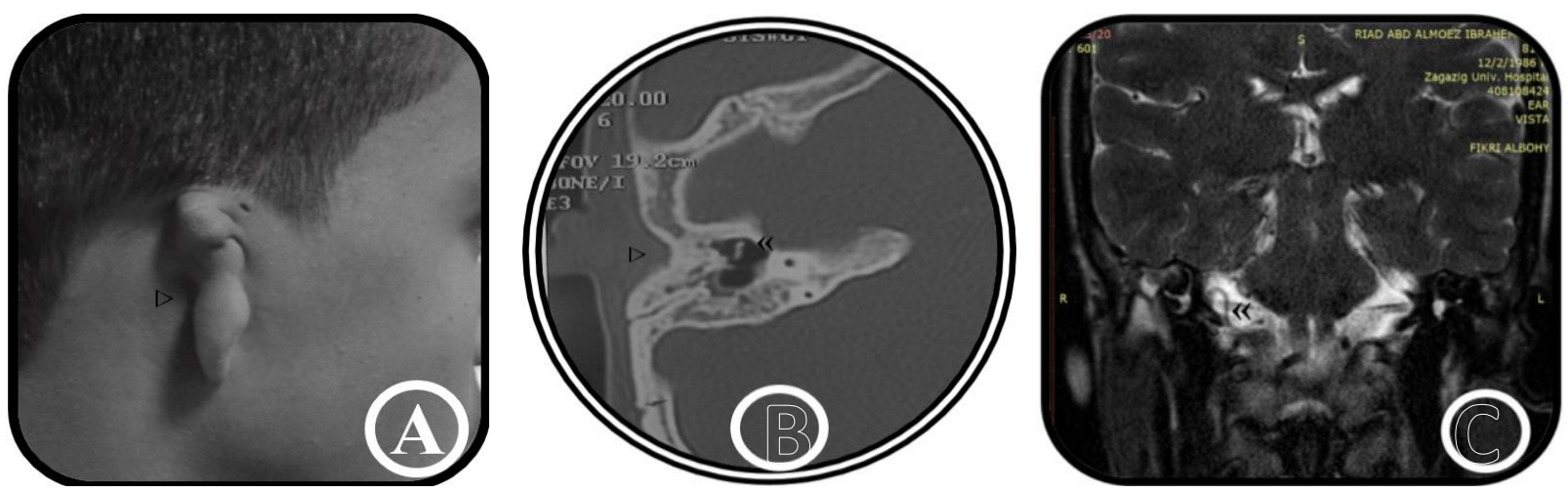

Case (2): A male patient 14 years old, with unilateral hear hearing loss, deformed right ear pinna, complain from tinnitus. (A) Black and white photograph: shows severe degree deformed right ear auricle $(D)$ and absent opening of the (EAC). (B) Magnified panels from axial CT images on right temporal bone: shows thick bony plate $(\triangleright)$ of the right (EAC), the tympanic cavity seen partially filled with fluid, deformed malleus and incus bones, displaced superiorly into the epitympanum, fused to each other («) with poor pneumatization of the right mastoid air cells. (C) Coronal images from b-FFE MRI of the both cerebellopontine angles: Reveal normal cochlear turns and configuration with normal cochlear nerves at both internal auditory canals till the cochlear apertures. A right cerebello-pontine angle signal void vascular structure («) originating from the basilar artery, anterior inferior cerebellar artery (AICA), passing in loop shaped near the right internal auditory canal.

The abnormal intensity at the middle ear cavity in (2) patients demonstrated as low signal intensity on T1WI and high signal intensity on T2WI representing fluid effusion. The ossicular chains are found as high signal intensity in (2) patients. In (4) patients, abnormal intensities in the mastoid cavity demonstrate intermediate signal intensities on both T1-and T2- weighted images.

\section{II- Sensineural hearing loss: -}

A twenty three patients diagnosed as having sensineural hearing loss, (14) males and (9) females, their age ranged from 3 to 6 years with mean age of 3.8 years and $\mathrm{SD}=1.742$. The main complaint was hearing loss with abnormal hearing tests. Other dysmorphic features of cranio-facial anomalies as a part of a congenital syndrome were found in (3) patients (1 male and 2 females) were diagnosed as one male case of Down's syndrome, one female case with
CHARGE syndrome and one female case of Waardenberg's syndrome.

Audiometric data were available in all patients, from different degrees of bone conduction. Profound SNHL has been linked with normal HRCT and MRI imaging in (10) patients (8 males and 2 females). According to case histories, all patients suffered from HL since birth and early childhood, the course of hearing was known from case history in all the patients as seen in Table (3) and Chart (3)

\section{(A) Cochlear abnormalities: (Case 3)}

According to HRCT, cochlear shape and size were detected in both axial and coronal scan. Further image reconstruction were done in multiplaner reformates (MPR) in short axis (Poschl) projection and long axis (Stenvers) projection were used. A normal cochlea in (19) patients, was diagnosed when there were 2 and $1 / 2$ turns and modiolus. In one female patient with osseous 
spiral lamina is absent and middle and apical turns coalesce to form a cystic apex but basal turn appears normal, was diagnosed as Mondini deformity, cochlear hypoplasia is seen in one female case with CHARGE syndrome, another male case with bilateral cystic common cavity the osseous spiral lamina is absent in all the cochlear turns, coalesce to form a cystic common cavity with the vestibule.

In MRI study, the Mondini deformity an additionally a visible basilar membrane separating the scala vestibuli and tympani into two chambers of same size. The female patient with CHARGE syndrome, the cochlea on both sides are hypoplastic with reduced turns and low fluid membranous signal, she preclude from cochlear implant. In the male patient with common cavity, the fluid signal of the membranous labyrinth were confirmed by MRI with b-FFE series, was done to evaluate the appearance of featureless cochlear cystic cavity, the later patient is scheduled for cochlear implant.

\section{(B) Cochlear nerve abnormalities:}

In MRI study, the axial, coronal and para-sagittal b-FFE series, were done to evaluate the appearance of the nerve bundles in the internal auditory canal, there are two male and female patients, diagnosed to have cochlear nerve hypoplasia and preclude for cochlear implant. The facial nerve in both patients was normal with no clinical manifestation for facial palsy. The appearance of the cochlear nerve in relation to the nerve bundles in the internal auditory canal seen in (21) patients, satisfactory definition of the nerve bundle in the background of the cerebrospinal fluid signal are further compared to various MRI parameters, where it is best assessed in the coronal and para-sagittal b-FFE series, as shown in Table (3) and Chart (3).

\section{(B) Malformations of the Semicircular Canals:} According to HRCT and MRI, the horizontal semicircular canal were dysplastic in (5) patients ( 2 males and 3 females), and the posterior semicircular canal was dysplastic in a female patient with Waardenberg's syndrome. The superior semicircular canal bony dehiscent is seen in a male patient detected in coronal scan. Further reformatted CT image of the temporal bone in the $45^{\circ}$ oblique plane reconstruction in the plane of the canal shows different widths of the bone cover, which decreases along curve of the roof of the superior semicircular canal. The MRI appearance of the semicircular canal dysplasia is best assessed in the 3D b-FFE as summarized in Table (3) and Chart (3). A male patient (2 months) with congenital hearing loss diagnosed as Down's syndrome, on the HRCT there was superior semicircular canal dysplasia.

(C) Enlarged Vestibular Aqueduct: (Case 4)

An enlarged vestibular aqueduct, was the most common inner ear malformation found in our study (8) patients, with (11.6\% of all SNHL), its considered as enlarged when caliber was more than $>1.8 \mathrm{~mm}$ measured at the midpoint between the common crus and the external aperture. The (8) patients underwent HRCT and MRI, in which the vestibular aqueduct was enlarged on CT $(>1.8$ $\mathrm{mm}), \mathrm{CT}$ and MRI yielded the same diagnostic results. The diameter of the vestibular aqueduct and endolymphatic duct ranged from 1.8 to 2.6 $\mathrm{mm}$ (mean: $2.1 \mathrm{~mm}$ ). A comparison between measurements in the same individual showed a wider vestibular aqueduct compared with the endolymphatic duct with exception of one ear, the endolymphatic structures were visible enlarged in MRI series at the axial b-FFE as summarized in Table (3) and Chart (3).

(D) Internal auditory canal :-

According to the HRCT, reveal marked reduction in the size of the internal auditory canal near the cochlear aperture in (2) patients (male and female) and diagnosed as stenotic internal audioty canal and were diagnosed by MRI to have cochlear nerve hypoplasia.

According to the MRI, the width and the cerebello-spinal fluid signal of the internal auditory canal of the (23) patients are seen in the coronal and para-sagittal b-FFE series, give the same results as the HRCT, as summarized in Table (3) and Chart (3).

III. Mixed hearing loss: -

Five patients, diagnosed as having mixed hearing loss. Vertigo and tinnitus is present in (3) patients. Audiometrically, (4) patients, were cooperative enough to complete both air and bone conduction behavioral audiometry. The conductive component was the predominant in all cases. In HRCT, two male patients with right external auditory canal atresia, one female patient with incudo-malleolar sublaxation and two females with hypoplastic middle ear cavity. In the MRI, preserved signal intensity of the membranous labyrinth with no congenital abnormalities seen in the inner ear structures.

Pre-operative cochlear implant: (Case 5)

In our study, (14) patients were prepared for cochlear implant surgery (8 males and 6 females), their age ranged from $3-6$ years with mean age of 4.2 years $\mathrm{SD}=0.978$. 
Table (3) Anatomic description of the deformed and other malformed parts of inner ear

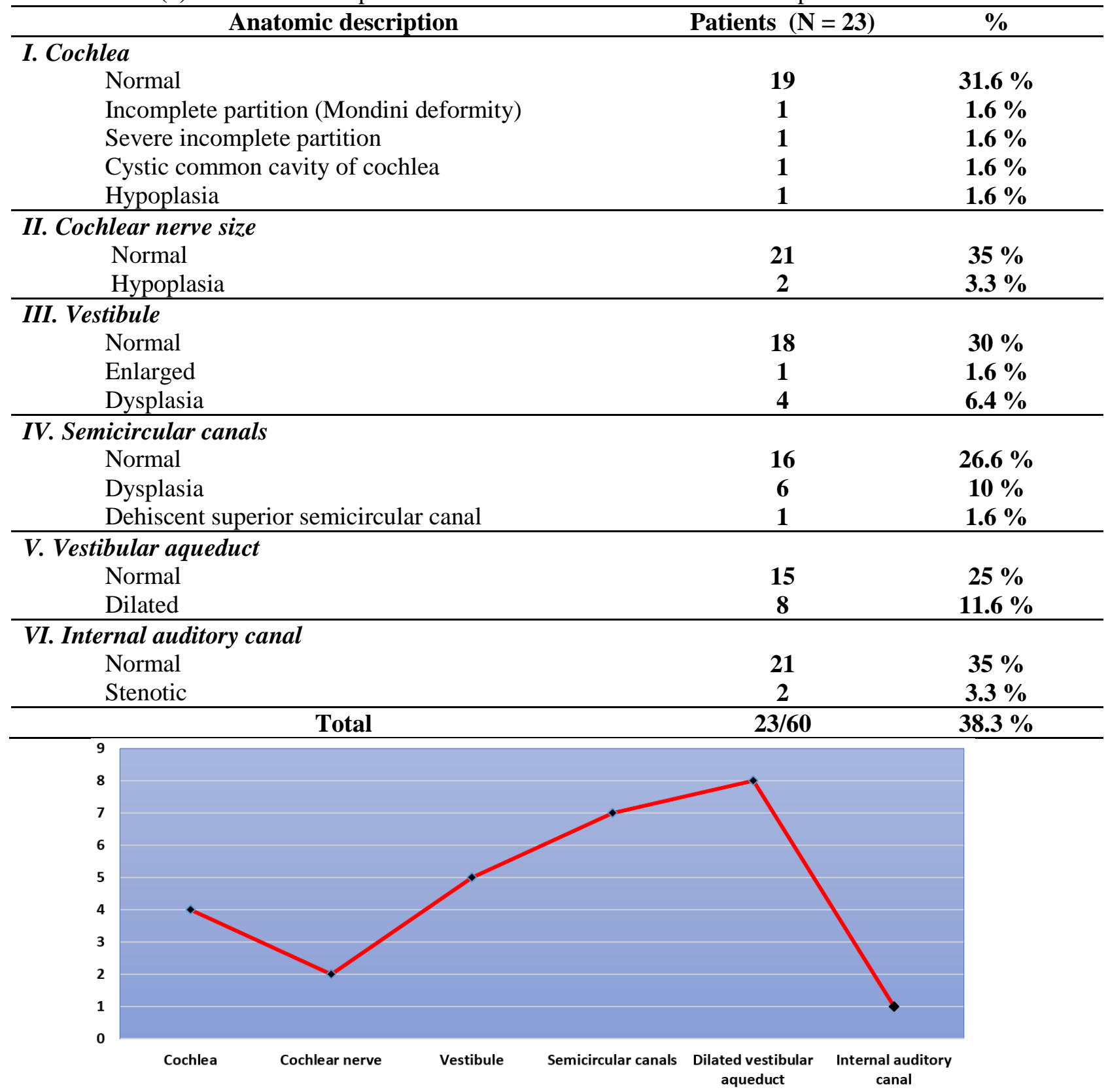

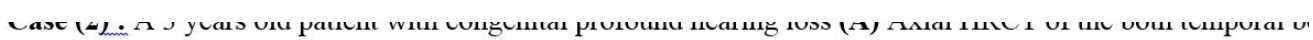

bilateral enlarged aqueduct syndrome. (B) The para-sagittal b-FFE of the right ear, demonstrate large vestibular : endolymphatic sac.
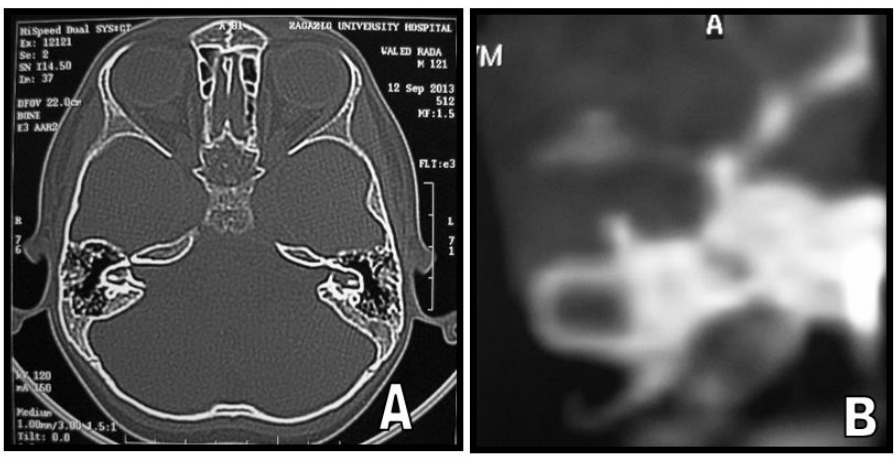

Chart (3): Line chart reveals the number of patients with deformed inner ear structures. 
Case (3): A 4 years old patient with congenital profound hearing loss (A) Axial HRCT of the both temporal bones of with bilateral enlarged internal auditory canal, cystic dilatation of the cochlea with featureless architectures. (B) MRI 3D b-FFE of the right inner ear, demonstrate common cavity signal with cystic dilatation of the cochlear and vestibule.
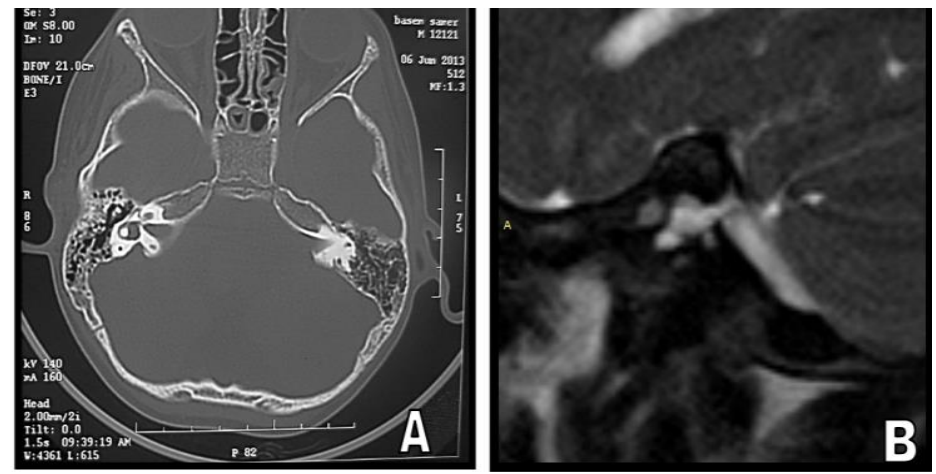

se (2): A 3 years old patient with congenital profound hearing loss (A) Axial HRCT of the both $t$

Case (4): A 3 years old patient with congenital profound hearing loss (A) Axial HRCT of the both temporal bones of with bilateral enlarged aqueduct syndrome. (B) MRI of the para-sagittal b-FFE at the right cerebello-pontine angle (CPA), demonstrate large vestibular aqueduct with endolymphatic sac.

All patients are having congenital sensineural hearing loss, assessed by HRCT and MRI of the temporal bones with no imaging abnormalities in (10) patients and (3) patients with congenital inner ear malformations, one male patient with bilateral common cystic cavity, another two male patients with bilateral enlarged vestibular aqueduct and endolymphatic sac, and the last patient was a female with Waardenberg's syndrome.

HRCT detect mastoid pneumatization in all of the patients and the second most common findings was high riding jugular bulge. The position of the tympanic segment of the facial nerve in the facial recess was declared (7) patients. The size of the cochlear nerve in the internal auditory canal is assessed in para-sagittal b-FFE in comparison to the facial, superior and inferior divisions of the vestibular nerves, patency of cochlear lumen, the size of the basal turn and bony modiolus are assessed by HRCT and further MRI of the cerebello-pontine angles with 3D b-FFE were done.
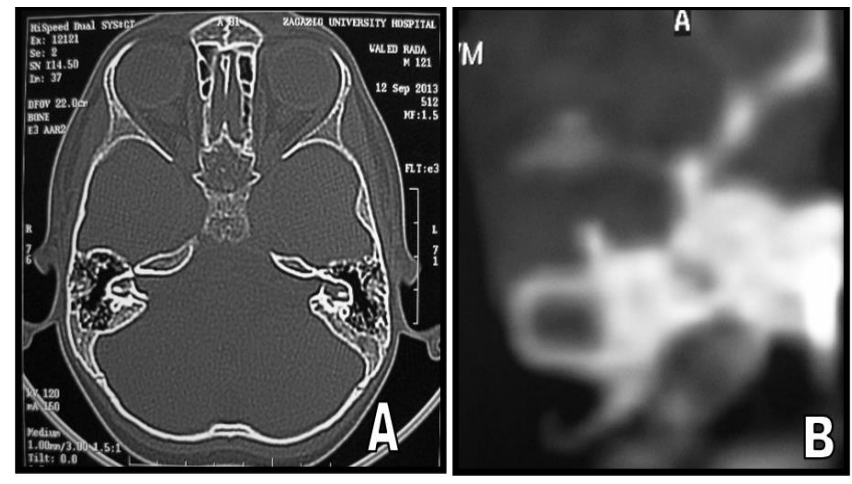

\section{Post-operative cochlear implant: (Case 6)}

Four patients followed up after cochlear implant, ( 2 males and 2 females) their ages range from 3.56 years. A three patients ( 2 males and one female) with trans-mastoidectomy and posterior tympanotomy approach and one female patient with right supra-meatal approach. All have $24 \mathrm{hrs}$ post-operative digital radiography image by Stenver's view to show the antenna with magnet, receiver-stimulator, and electrode array coiled within the cochlea. High-resolution coronal images of the temporal bone have demonstrate the entire labyrinthine path of the electrode array within the cochlear basal turn with no evidence of malposition. One case (supra-meatal approach), has soft tissue granulation density in the middle ear cavity and external ear. 
Case (5) : A 4 years old patient with congenital profound hearing loss (A) Axial HRCT of the both temporal bones of with bilateral enlarged internal auditory canal, cystic dilatation of the cochlea with featureless architectures. (B) The 3D b-FFE of the right ear, demonstrate common cavity signal with cystic dilatation of the cochlear and vestibule.

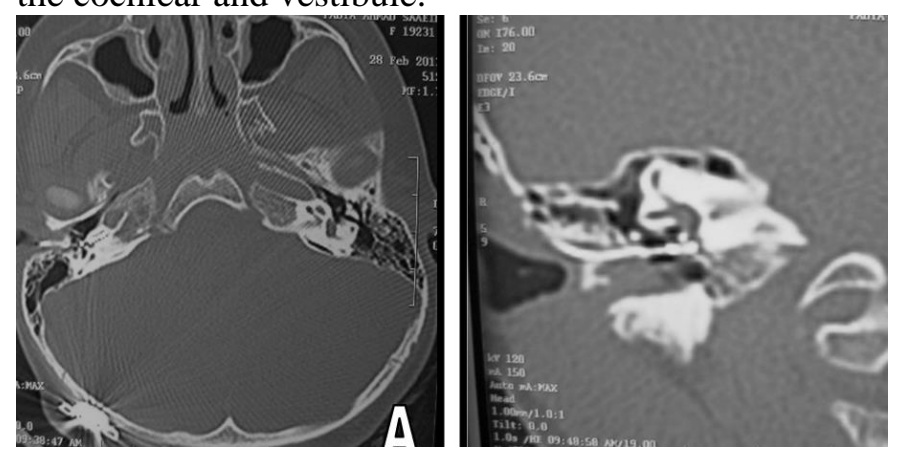

Case (6) : A 5 years old female patient with post (A) Axial CT images of the both temporal bone: Reveal the magnet and electrode array passing though a tunneling at the right external auditory canal, till it is positioned with its internal channels seen at the basal turn of the cochlea with a soft tissue density mass filling the right external auditory canal surrounding the internal electrode, abutting the tympanic membrane without involvement of the middle ear (B) Magnified coronal CT images of the right temporal bone: Reveal the inflammatory soft tissue at the external auditory canal surrounding the internal electrode and abutting the tympanic membrane with intact aero-tympanic cavity and pneumatization of the mastoid antrum.

\section{DISCUSSION}

The availability of clinical data and history are positively correlated with the correct diagnosis of congenital hearing loss. However, in patients presenting at various age, they may forget various aspects of the clinical history, which could compromise the aetiological investigation. This study illustrates and clearly justifies the need to perform a standardized aetiological work-up, including a standardized clinical interview, and first-line of investigations are similar for the two types of congenital hearing loss and comprise systematic clinical interview, clinical examination, family history, ophthalmological examination, petrous temporal bone imaging, serology are systematically done in children with severe or profound hearing loss.

Gibbin, $2008{ }^{(5)}$ Agree that most important element in the diagnosis of the cause of deafness in any child with a demonstrated hearing loss is the history, although clearly other investigations may also prove highly important. The history should be targeted to three key areas, pre-natal including family history, perinatal and post-natal. A full clinical examination is essential, although the majority of children with congenital hearing loss will show no related clinical abnormality.

Joshi et al., $2012{ }^{(6)}$ Clear understanding of the embryogenesis and anatomy of the external, middle and inner ear and the standard classification systems for describing inner ear malformations are fundamental to the interpreta- tion of CT and MR. Identification of the ear abnormality has a substantial effect on clinical decision making, prognosis, and management strategy.

On the basis of this study, imaging provides vital information about ear malformations and forewarns the surgeon to the possibility of potential complications and guides parental expectations during preoperative counseling.

Thomeer, $2012{ }^{(7)}$ A variety of anomalies challenge the experienced surgeon, requiring different reconstruction strategies to improve the patient's hearing level. Preoperative assessment is mandatory to search for syndromal diagnoses, which might be important for patient counseling and prognosis.

Since HRCT restricts the plane of imaging in temporal bone to the standard axial and coronal orientations, the use of high resolution multi-planar reformat (MPR) from conventional high resolution CT image data can be rotated in space and dissected in any plane, for demonstrating spatial relationships between structures in the malformed ear and for building an individual spatial image of the patient while doing the operation. We reproduced common MPR section planes on short and long axis of the cochlea. It was demonstrated that the ossicular chain is arranged obliquely in common MPR sections. Moreover, we demonstrate a method for sequential reformation of four section planes that 
is optimal for observing the entire cochlea in two planes.

Elziere et al., $2012^{(8)}$ Petrous temporal bone imaging is one of the most useful investigations to establish an aetiological diagnosis of hearing loss. However, these examinations are performed in some hospitals, including our own, under general anaesthesia for children under the age of 4 years, or because of the clinician's refusal or omission to propose this examination.

Kadom and Sze, $2010{ }^{(9)}$ CT of the temporal bones is a valuable tool in the diagnosis and treatment of pediatric congenital hearing loss and the key functions of the radiologist are design of optimal imaging studies for children and indepth anatomic-pathologic knowledge for preand postsurgical anatomy.

Veillon et al., $2008^{(10)}$ The temporal bone and adjacent regions can be evaluated in detail by CT and MRI, which not only provide important diagnostic information but also insight into pathological procedures and their extension within these structures. To benefit from these technologies, the radiologist and clinician knowledge of the anatomy of this region is absolutely necessary.

Auricular malformations can affect the size, shape, position, and orientation of the pinna. Various classification schemes have been proposed to grade the severity of deformity; however, Weerda's classification is most widely used. This classification includes 3 grades of microtia with worsening severity.

Abowarda and Nasr, $2010{ }^{(11)}$ Microtia is commonly associated with a variety of external, middle, and, less frequently inner ear abnormalities. High-resolution multiplanar CT scanning is the primary imaging modality of choice in the preoperative evaluation of patients with microtia and external auditory canal dysplasia.

Our MRI studies were unable to evaluate the condition of the middle ear and mastoid cavity, which appears as intermediate and bright signals were seen in both $\mathrm{T} 1$ - and T2-weighted images. However, HRCT clearly shows the condition of the tympanic cavity and mastoid aerated cells clearly.

Shraddha et al., $2011^{(12)}$ Middle ear malformations can affect the normal development of the tympanic cavity, mastoid pneumatization, and ossicles. There are various classifications proposed, so it is important that the radiologist and the otologist use the same or similar classification to evaluate middle ear anatomy.
The most common middle ear anomalies in our study were anomalies of ossicles and related structures as fixation of the incudomalleolar joint, bony fusion of the head of the malleus to the long process of the incus. According to HRCT findings; it was superior in detection of bony landmarks of the temporal bones. These findings were the shape and orientation of the petro-temporal bones, the patency of the external auditory canal (bony atresia), associated tympanic cavity and ossicular chain anomaly.

Huang et. al, $2012^{(13)}$ SNHL presenting later in life is often related to inner ear malformations any of which may be suggested by findings at cross-sectional imaging. Neuroradiologists who routinely interpret temporal bone studies performed for the evaluation of childhood SNHL should be familiar with these potential causes and their respective imaging appearances; furthermore, they should be familiar with findings such as severe labyrinthine ossification, which might preclude or complicate cochlear implantation in a child with hearing loss.

Gupta, 2010 (14) Imaging plays an important role in diagnosis and work-up of patients with SNHR. There are a whole lot of etiologies for SNHR, many of which have typical findings on HRCT and MRI, which help in differentiation of these causes and therefore guides the treating surgeon in further management. Both CT and MRI are complimentary to each other and should be judiciously used by the radiologist in diagnosing the various congenital malformations discussed.

Enlarged vestibular aqueduct is one of the most frequently encountered inner ear malformations in our study, all cases were bilateral and they presented with progressive profound sensorineural deafness, the diameter of the vestibular aqueduct is compared with the diameter at the mid portion of the adjacent SCC as measured in the axial HRCT or the diameter of the vestibular aqueduct is larger than the SCC, a diagnosis of enlarged vestibular aqueduct. As seen in the MRI the enlarged endolymphatic duct and sac is linked with SNHL in one patient.

MR imaging is superior to CT for the identification of an enlarged vestibular aqueduct. The additional acquisition of axial T2-weighted MR images of the brain during imaging of the inner ear allows the identification of brain abnormalities that commonly coexist with congenital inner ear malformations ${ }^{(6)}$.

Krombach et al., $2008{ }^{(15)}$ MRI and HRCT have become essential parts of the 
diagnostic work-up in patients with symptoms related to the inner ear. Both imaging modalities are complementary. The strength of CT lies in the better delineation of the osseous otic capsule. The soft-tissue components cannot be evaluated. The fluid-containing labyrinth, the nerves and softtissue components can be excellently delineated on MR images. According to the imaging modality must be chosen. Both modalities are applicable for the delineation of malformations. In patients for whom cochlear implants are planned, MRI and CT should be part of the preoperative work-up.

The study has classified inner ear malformations according to embryogenesis, and this classification is widely accepted as an initial systematic basis for the categorization. Although our sample size is not large, radiological findings of congenital cochlear malformations in the present study two different types incomplete partition IP-II (the classic Mondini deformity). Classic Mondini deformity has three components (a cystic apex), whereas the IP-I malformation with cystic cochleovestibular malformation, one patient with bilateral common cavity, another patient with cochlear hypoplasia. In this way we group these complex malformations and compare the results of appearance in various MRI parameters.

Incesulu et al., $2002{ }^{(16)}$ Inner ear malformations form a heterogeneous group ranging from complete aplasia of the inner ear to dysplasia of one anatomic region. Since Mondini first presented his findings of combined membranous and bony dysplasia of the inner ear based on anatomical dissection, many investigators have documented a variety of inner ear malformations.

Some workers have attempted to establish normative cochlear measurements in normal as well as abnormal inner ears on HRCT. As they believe that measurements can increase the pick up rate of subtle inner ear abnormalities. However, we found that the acquisition and comparison of these measurements was time consuming and did not influence decision making for cochlear implantation. Therefore we did not include them in our protocol.

Natacha et al., $2010{ }^{(17)}$ A radiological checklist for the pre-cochlear implant assessment of children with profound hearing loss, performing a complementary MRI when the width of the bony canal for the cochlear nerve is $<2 \mathrm{~mm}$. This should help avoid implantation of children with profound SNHL due to an absent cochlear nerve. However, several children without any hearing loss had measurements between 1.7 and 2 $\mathrm{mm}$. We cannot, therefore, base our SNHL diagnosis solely on this measurement; clinical data and an audiogram are essential. In the particular case of pre-cochlear implant assessment, MRI is mandatory whatever the size of the canal to confirm the integrity of the nerve.

In our study, classification schemes to categorize inner ear malformations have been proposed based on radiographic findings, which divides malformations into categories based on a timeline of developmental arrest on cochlear involvement and includes: Common cavity deformities in which an absence of differentiation of the otocyst results in a common cochleovestibular chamber; Cochlear hypoplasia which describes varying degrees of cochlear development from a rudimentary diverticulum to a small cochlear bud; and incomplete partition which results from failure of development of the full intra-cochlear partition affecting the apical and middle turns of the cochlea, this deformity results in the classic Mondini's dysplasia and is often accompanied by other anomalies, such as a dilated vestibule and/or enlarged vestibular aqueduct.

Mc Elveen and Cunningham, $2010{ }^{(18)}$

The variations in inner ear malformations may influence the surgeon's technique for electrode placement in cochlear implant. For example, malformations without a well-formed intracochlear partition, such as common cavity malformations, tend to have the neuroepithelium located on the outer wall of the cavity. Consequently, the electrode array must be positioned to accommodate this. In addition, the surgeon must consider the associated facial nerve abnormalities and increased risk of perilymphatic/cerebrospinal fluid (CSF) leakage when planning the surgical approach. To minimize the unexpected and to maximize successful surgical outcomes in this patient population, it is imperative that a thorough preoperative evaluation be undertaken.

Although many of the syndromes associated with congenital hearing loss do not usually demonstrate gross inner ear anomalies by imaging, the presence of specific inner ear anomalies is characteristic of certain syndromessuch as in the case of CHARGE syndrome presented with bilateral cochlear dysplasia, chochlear nerve aplasia .

Robson, $2006{ }^{(19)}$ There have been major advances in the characterization of the genetic of congenital deafness. This provides the opportunity to correlate imaging findings in patients with 
established etiologic diagnoses. In some cases, typical imaging findings can provide clues to a diagnosis that had not been suspected, such as the characteristic cochlear anomalies seen in the inner ear, nasal and ocular findings in the CHARGE syndrome.

Most patients with profound sensorineural hearing loss due to inner ear malformations are suitable for cochlear implantation. The surgical approach selected is based on the type of malformation and the radiographic findings. Although the trans-mastoidectomy with posterior tymapnotomy approach is the approach most commonly used, the supra-meatal approach is preferred in cases where the facial nerve may take an aberrant course.

Kim et al., $2006^{(20)}$ All children with inner ear malformations can make substantial improvements in their speech recognition after implantation. Even severe forms of malformation, such as a common cavity and severe cochlear hypoplasia, have been associated with good results. In cases of a narrow IAC, implantation can be considered carefully if a functioning auditory nerve is demonstrated radiologically and audiologically. The parents must be counseled thoroughly and must have appropriate expectations before implantation is undertaken, because the individual outcomes after implantation range all the way from achievement of open-set speech perception to failure of auditory perception.

Robert et al., $2003{ }^{(21)}$ Future imaging of implant candidates include a routine evaluation of the facial nerve, IAC and cochlea, and objective measurements of cochlear nerve diameter may prove to be of prognostic value in determining the potential benefit of a cochlear implant.

\section{CONCLUSION}

Imaging plays an important role in diagnosis and work-up of patients with congenital hearing loss. There are a whole lot of etiologies, many of which have typical findings on HRCT and MRI, which help in differentiation of these causes and therefore guides the treating surgeon in further management. Both HRCT and MRI are complimentary to each other and should be judiciously used by the radiologist in diagnosing the various congenital malformations.

\section{REFERENCES}

(1) Canale A., Favero E., and Lacilla M. (2006): Age at diagnosis of deaf babies: a retrospective analysis highlighting the advantage of newborn hearing screening. Int J Pediatr Otorhinolaryngol, 70:1283-9.
(2) Angeles E., David O. and Gareth W. (2006): The diagnosis of hearing loss in children: Common presentations and investigations. Current Paediatrics, 16:484-488.

(3) Mikulec A. (2009): Congenital Hearing Loss (Sensorineural and Conductive), Pediatric Otolaryngology for the Clinician, Part 2: 7579.

(4) Adrienne M. L., Sarah C., Sarah M. and John A. G. (2009): Etiology of unilateral neural hearing loss in Children. International Journal of Pediatric Otorhinolaryngology, 73, 417-427.

(5) Gibbin K. P., (2008): Pediatric ENT, Management of the Deaf Child, Springer Copyright (C) by Sprinter, Inc.

(6) Joshi V.M., Navlekar S.K., Kishore G.R. et al., (2012): CT and MR Imaging of the Inner Ear and Brain in Children with Congenital Sensorineural Hearing Loss, radiographics.rsna.org May-June 2012: 683698.

(7) Thomeer H. G., Kunst H.P. and Cor Cremers W.R. (2012): Congenital Ossicular Chain Anomalies Associated With a Mobile Stapes Footplate: Surgical Results for 23 Ears, Annah of Otology. Rhinology \& Laryngology; 121(4):275-281.

(8) Elziere M., Roman S., Nicollas R. et al., (2012): Value of systematic aetiological investigation in children with sensorineural hearing loss, European Annals of Otorhinolaryngology, Head and Neck diseases; (129):185-189.

(9) Kadom N. and Sze R.W. (2010): Radiological Reasoning: Congenital Sensorineural Hearing Loss, AJR; (194): Sep;89 (9):733-7.

(10) Veillon F., Riehm S., Abu Eid M. et al., (2008): Imaging of Temporal Bone Pathology, Diseases of the Brain, Head \& Neck, Spine 2008, Part 1, 141-148.

(11) Abowarda M.H. and Nasr W.F. (2010): Patients with dysplasia of the auricle and external ear abnormalities: Evaluation of the temporal bones malformation with thin-section computed tomography, The Egyptian Journal of Radiology and Nuclear Medicine; (41): 453-458. (53).

(12) Shraddha S.M., Hemant A. P. and Mohannad I. (2011): Congenital Malformations of the Temporal Bone. Neuroimag Clin N Am; (21): 603-619.

(13) Huang B.Y., Zdanski C. and Castillo M., (2012): Pediatric Sensorineural Hearing Loss, Part 1: Practical Aspects for Neuroradiologists. AJNR Am J Neuroradiol; (33): 211-217. 
(14) Gupta S., (2010): Otorhinolaryngology Clinics: Imaging for Sensorineural Hearing Loss, An International Journal, May-August; 2(2):113-124.

(15) Krombach G.A., Honnef D., Westhofen M. et al., (2008): Imaging of congenital anomalies and acquired lesions of the inner ear, Eur Radiol; (18): 319-330.

(16) Incesulu A., Vural M., Erkam U. et al., (2002): Cochlear implantation in children with inner ear malformations: report of two cases. International Journal of Pediatric Otorhinolaryngology; (65): 171-179.

(17) Natacha T., Thierry V. D., Monique E. et al. (2010): Computed tomography measurements of the normal and the pathologic cochlea in children, Pediatr Radiol, (40):275-283.

(18) Mc Elveen J.T. and Cunningham C.D. (2010): Cochlear implantation in the congenitally malformed ear, Operative Techniques in Otolaryngology; (21): 243-247.

(19) Robson C.D., (2006): Congenital hearing impairment, Pediatr Radiol (36): 309-324.

(20) Kim L.S., Jeong S.W., Huh M.J. et al., (2006): Cochlear Implantation in Children With Inner Ear Malformations, Annals of otology, rhinology \& Laryngology; 115(3):205-214.

(21) Robert J. W., John I. L., Colin L.W. et al., (2003): Pediatric and Adult Cochlear Implantation, RadioGraphics; 23:1185-1200. 